\title{
Hexapod coloron at the LHC
}

\author{
Yang Bai, ${ }^{a}$ Sida $\mathbf{L u}^{a}$ and Qian-Fei Xiang ${ }^{a, b}$ \\ ${ }^{a}$ Department of Physics, University of Wisconsin-Madison, \\ Madison, WI 53706, U.S.A. \\ ${ }^{b}$ Key Laboratory of Particle Astrophysics, Institute of High Energy Physics, \\ Chinese Academy of Sciences, \\ Beijing 100049, China \\ E-mail: yang.bai@wisc.edu, slu57@wisc.edu, xiangqf@ihep.ac.cn
}

ABSTRACT: Instead of the usual dijet decay, the coloron may mainly decay into its own "Higgs bosons", which subsequently decay into many jets. This is a general feature of the renormalizable coloron model, where the corresponding "Higgs bosons" are a color-octet $\Theta$ and a color-singlet $\phi_{I}$. In this paper, we perform a detailed collider study for the signature of $p p \rightarrow G^{\prime} \rightarrow(\Theta \rightarrow g g)\left(\phi_{I} \rightarrow g g q \bar{q}\right)$ with the coloron $G^{\prime}$ as a six-jet resonance. For a light $\phi_{I}$ below around $0.5 \mathrm{TeV}$, it may be boosted and behave as a four-prong fat jet. We also develop a jet-substructure-based search strategy to cover this boosted $\phi_{I}$ case. Independent of whether $\phi_{I}$ is boosted or not, the $13 \mathrm{TeV}$ LHC with $100 \mathrm{fb}^{-1}$ has great discovery potential for a coloron with the mass sensitivity up to $5 \mathrm{TeV}$.

Keywords: Beyond Standard Model, Technicolor and Composite Models

ARXIV EPRINT: 1805.09815 


\section{Contents}

1 Introduction 1

2 A short summary of the ReCoM 3

3 Productions and new signatures at the LHC 5

4 Search strategies and estimated sensitivities $\quad 6$

$\begin{array}{lll}4.1 & \text { Six-jet resonance search for coloron } & 7\end{array}$

$\begin{array}{ll}4.2 \text { Coloron as a resonance containing a fat jet } J_{\phi_{I}} & 11\end{array}$

5 Discussion and conclusions 13

$\begin{array}{lll}\text { A } \tau_{4} / \tau_{3} \text { distribution for the fat } \phi_{I} \text { jet } & 14\end{array}$

\section{Introduction}

The renormalizable coloron model (ReCoM) [1, 2] is one of simplest gauge extensions of the Standard Model (SM). Due to the coloron's flavor-blind nature and orthogonality to the electroweak sector, its mass could be at the $\mathrm{TeV}$ scale and consistent with various experimental constraints. Therefore, the coloron is an ideal particle for the Large Hadron Collider (LHC) to search for. Besides its phenomenological motivation, the coloron, or its cousin "axi-gluon", has been predicted in many scenarios beyond the SM [3-10] (see also [11-17] for related phenomenological studies).

At the LHC, the simplest signatures of colorons include dijet and $t \bar{t}$ resonances. Based on narrow dijet resonance searches of the $13 \mathrm{TeV}$ LHC with around $36 \mathrm{fb}^{-1}$ data, both CMS [18] and ATLAS [19] collaborations have set impressive constraints on the coloron mass to be above around $6 \mathrm{TeV}$ at $95 \%$ confidence level (CL). The constraints from $t \bar{t}$ resonance searches are weaker $[20,21]$. For both existence searches, the coloron is assumed to decay $100 \%$ into two quarks, which may be a too-simplicity assumption. As analyzed in refs. [1, 2], additional scalar bosons exist in the spectrum for a perturbative theory. This is a universal behavior of spontaneous breaking of renormalizable and perturbative gauge theory, for which one or more scalar bosons become the company of the massive gauge boson. The famous example is for sure the SM Higgs boson as a bi-product of the Higgs mechanism to explain $W$ and $Z$ gauge boson masses.

More specifically and in ReCoM, a minimal scalar field, that is bi-fundamental under the $\mathrm{SU}(3)_{1} \times \mathrm{SU}(3)_{2}$ gauge symmetry, develops a non-zero vacuum expectation value (VEV) to break the gauge symmetry to the $\mathrm{QCD} \mathrm{SU}(3)_{c}[16]$. There are three scalar bosons in the spectrum, $\phi_{R}, \phi_{I}$ and $\Theta$, with the first two color-singlet and the last one color-octet. More 

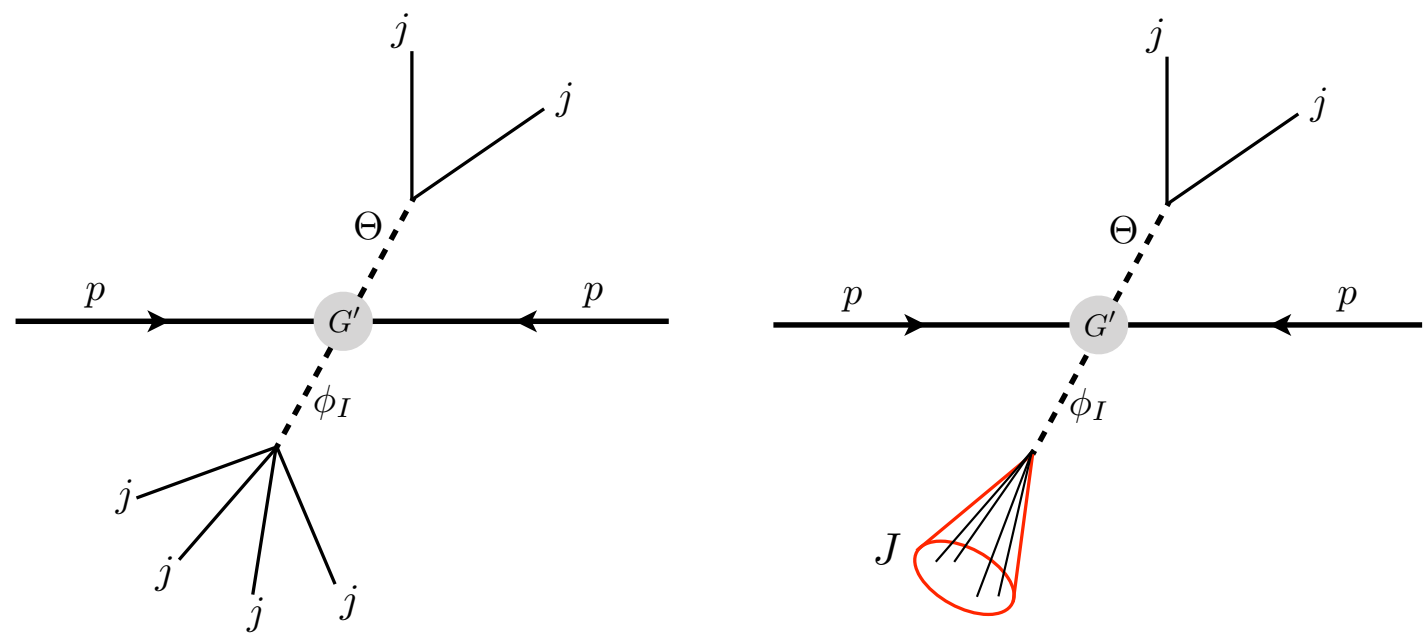

Figure 1. Left panel: the schematic plot for the coloron signature at the LHC as a six-jet resonance when $M_{\phi_{I}} \sim M_{G^{\prime}}-M_{\Theta}$. Right panel: when $M_{\phi_{I}} \ll M_{G^{\prime}}-M_{\Theta}$, the particle $\phi_{I}$ is boosted such that it may behave as a four-prong fat jet.

interestingly, there is spontaneous breaking of some enhanced global symmetries such that both $\phi_{I}$ and $\Theta$ could be pseudo Nambu-Goldstone Bosons (PNGB's) and naturally lighter than the coloron $G^{\prime}$. As a result, there are two additional decay channels for the coloron, $G^{\prime} \rightarrow \Theta \Theta$ and $G^{\prime} \rightarrow \Theta \phi_{I}$, which could have decay branching fractions larger than the one of coloron decaying into two quarks. There are two consequences for those additional decay channels. The first one is that the dijet constraints on the coloron masses could be dramatically relaxed and a coloron below $3 \mathrm{TeV}$ can still survive the current experimental searches. The second one is that there are additional opportunities for the LHC to discover the massive coloron via other signatures, which will be the main focus of this paper.

Depending on how $\Theta$ and $\phi_{I}$ decays, a high multiplicity of quarks and gluons or jets after hadronization are anticipated in the final state from the coloron's cascade decays. For instance, $\Theta$ can decay into two gluons at one loop or decay into $\phi_{I}$ plus $q \bar{q}$ at tree-level via an off-shell coloron. The decays of $\phi_{I}$ are more complicated. For a heavier $\phi_{I}$ above around $500 \mathrm{GeV}$, its main decay channel is a four-body one, $\phi_{I} \rightarrow g g q \bar{q}$, happening at one-loop. For a lighter $\phi_{I}$, its main decay channels are into two electroweak gauge bosons at three-loop and with a displaced vertex. In ref. [2], a throughout list of possible, $\mathcal{O}(50)$, final states has been tabulated. In this paper, rather than making an extensive study of all possible signatures, we mainly concentrate one particular decay chain $p p \rightarrow G^{\prime} \rightarrow(\Theta \rightarrow g g)\left(\phi_{I} \rightarrow q \bar{q} g g\right)$ and perform a detailed collider simulation to estimate the discovery potential at the $13 \mathrm{TeV}$ with $100 \mathrm{fb}^{-1}$.

Even after we fix the decay chain and depending on the mass spectra, there are still two possible different collider signatures. For the mass spectrum with $M_{\phi_{I}} \sim M_{G^{\prime}}-M_{\Theta}$, the $\phi_{I}$ scalar from coloron decays is not boosted, so the four partons from $\phi_{I}$ decays are likely to form four isolated jets if one uses the ordinary jet-finding algorithm. Together with the two jets from $\Theta$ decays, the coloron $G^{\prime}$ behaves as a six-jet resonance with the schematic process shown in the left panel of figure 1. We will develop a bump search strategy for this 
new type of signature and estimate the LHC sensitivities on the model parameter. The second possibility relies on the PNGB nature of $\phi_{I}$, which could be much lighter than both $\Theta$ and $G^{\prime}$. If $M_{\phi_{I}} \ll M_{G^{\prime}}-M_{\Theta}$, the $\phi_{I}$ particle from coloron decays is likely to be boosted and the four partons from its decay will be collimated. For this case, if one chooses the jet-finding algorithm with a larger value of geometric size, the four partons from $\phi_{I}$ decays can be grouped into a single fat jet, see the right panel of figure 1 . We will also develop a jet-substructure-based analysis for this type of signatures.

Our paper is organized as follows. In section 2, we provide a short summary for the ReCoM including the decay branching fractions of all three relevant particles, $G^{\prime}, \Theta$ and $\phi_{I}$. We show the production times branching fractions for the signatures in section 3 . In section 4.1, we study a few kinematic variables to optimize the searches for the six-jet resonances, while in section 4.2 we perform a jet-substructure-based analysis for the case with a fat $\phi_{I}$ jet. We conclude our paper in section 5 .

\section{A short summary of the ReCoM}

As a simple extension of the SM, the ReCoM serves as a representative one for a class of models with a color-octet massive gauge boson. The ReCoM model extends the SM with an $\mathrm{SU}(3)_{1} \times \mathrm{SU}(3)_{2} \times \mathrm{SU}(2)_{W} \times \mathrm{U}(1)_{Y}$ gauge group and the following spontaneous breaking of the gauge symmetry

$$
\mathrm{SU}(3)_{1} \times \mathrm{SU}(3)_{2} \stackrel{\langle\Sigma\rangle}{\longrightarrow} \mathrm{SU}(3)_{c},
$$

with the bi-fundamental scalar field $\Sigma$ transforming as $(3, \overline{3}, 1,1)$ under the gauge group and developing a VEV from minimizing a potential. The massive gauge bosons, corresponding to the broken gauge generators, are called the "coloron", $G^{\prime}$. All SM quarks are charged in the fundamental representation of $\mathrm{SU}(3)_{1}$, such that the coloron couples to quarks in the flavor-blind way and there is no flavor-changing neutral current or stringent constraints from flavor physics.

Other than the massive coloron field, there are three more scalar particles: a coloroctet real scalar field $\Theta^{a}$ and two scalar singlets $\phi_{R}$ and $\phi_{I}$. The masses of heavy coloron and $\phi_{R}$ are largely determined by the symmetry breaking scale $f_{\Sigma}$, while $\Theta$ and $\phi_{I}$ could be lighter due to their PNGB nature [2]. As emphasized in ref. [2], lighter $\Theta^{a}$ and $\phi_{I}$ could dramatically modify decays of the coloron, suppress the coloron dijet decay branching fraction and allow a lighter coloron to be searched for at the LHC. If kinematically allowed, the two-body tree-level decay widths of the coloron have $[1,2]$

$$
\begin{aligned}
\Gamma\left(G^{\prime} \rightarrow j j\right) & =5 \Gamma\left(G^{\prime} \rightarrow t \bar{t}\right)=\frac{5 \alpha_{s}}{6} \tan ^{2} \theta M_{G^{\prime}}, \\
\Gamma\left(G^{\prime} \rightarrow \Theta \Theta\right) & =\frac{\alpha_{s}}{32 \tan ^{2} \theta}\left(1-\tan ^{2} \theta\right)^{2} M_{G^{\prime}}\left(1-\frac{4 M_{\Theta}^{2}}{M_{G^{\prime}}^{2}}\right)^{3 / 2}, \\
\Gamma\left(G^{\prime} \rightarrow \Theta \phi_{I}\right) & =\frac{\alpha_{s}}{72 \tan ^{2} \theta}\left(1+\tan ^{2} \theta\right)^{2} M_{G^{\prime}}\left[1-2 \frac{M_{\Theta}^{2}+M_{\phi_{I}}^{2}}{M_{G^{\prime}}^{2}}+\frac{\left(M_{\Theta}^{2}-M_{\phi_{I}}^{2}\right)^{2}}{M_{G^{\prime}}^{4}}\right]^{3 / 2} .
\end{aligned}
$$

In the first line of the above equation for the decays into quarks, we have summed the five light flavors and ignored the quark masses. The mixing angle, $\tan \theta$, is related to 

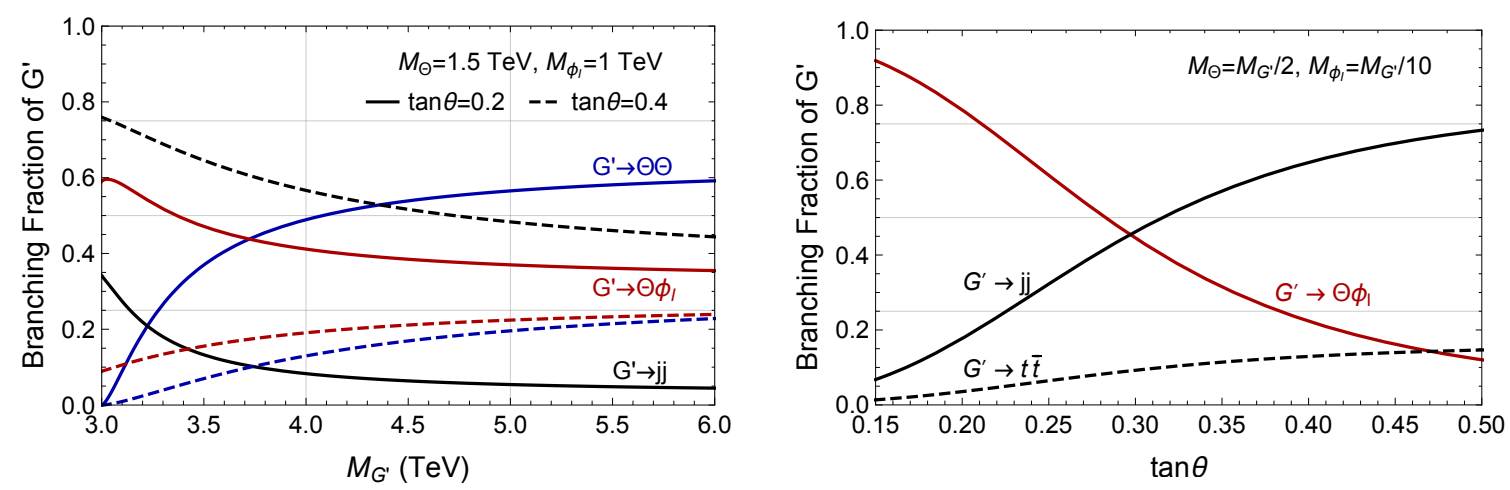

Figure 2. Branching fraction of $G^{\prime}$ as a function of $G^{\prime}$ mass or the mixing angle. In the left panel, the $\Theta$ and $\phi_{I}$ masses are fixed, while in the right panel their ratios over the coloron mass are fixed. The branching fraction of $t \bar{t}$ final state (not shown on the plot) is roughly $1 / 5$ of the $j j$ final state.

the quark couplings to coloron as $g_{s} \tan \theta \bar{q} \gamma^{\mu} T^{a} G_{\mu}^{\prime a} q$. So, for a small value of $\tan \theta$, the branching ratio for the coloron dijet decays is suppressed. Requiring the gauge couplings to be perturbative, the mixing angle is constrained to be $0.15 \lesssim \tan \theta \lesssim 6.7$.

Numerically, we show the branching fractions of coloron decays in figure 2 . In the left panel of figure 2, we fix the $\Theta$ and $\phi_{I}$ masses to be $1.5 \mathrm{TeV}$ and $1 \mathrm{TeV}$, respectively, while in the right panel we fix the mass ratios to be $M_{\Theta} / M_{G^{\prime}}=1 / 2$ and $M_{\phi_{I}} / M_{G^{\prime}}=1 / 10$. For the perturbative-allowed range of $\tan \theta$, the ratio of the coloron width over mass is below $5 \%$, so the coloron behaves as a narrow resonance at the LHC.

There are two main decay channels for the color-octet scalar $\Theta: \Theta \rightarrow g g$ and $\Theta \rightarrow q \bar{q} \phi_{I}$. From one-loop diagrams, its decay into two gluons has the form of

$$
\Gamma(\Theta \rightarrow g g)=\frac{15 \alpha_{s}^{2} \bar{\mu}_{\Sigma}^{2}}{128 \pi^{3} M_{\Theta}}\left(\frac{\pi^{2}}{9}-1\right)^{2}\left(1+r_{\mathcal{A}}\right), \quad \bar{\mu}_{\Sigma}=\frac{3 g_{s}\left(1+\tan ^{2} \theta\right)}{2 M_{G^{\prime}} \tan \theta}\left(M_{\Theta}^{2}-\frac{8}{9} M_{\phi_{I}}^{2}\right),
$$

with the parameter $r_{\mathcal{A}}$ given by

$$
r_{\mathcal{A}}=\frac{3}{32 \sqrt{2}\left(\pi^{2}-9\right)} \frac{\mathcal{A}\left[M_{\Theta}^{2} /\left(4 M_{G^{\prime}}^{2}\right)\right]}{1-8 M_{\phi_{I}}^{2} /\left(9 M_{\Theta}^{2}\right)}, \quad \mathcal{A}(\tau)=\left[2 \tau^{2}+3 \tau+3(2 \tau-1) \arcsin ^{2} \sqrt{\tau}\right] \tau^{-2} .
$$

Numerically, $r_{\mathcal{A}} \approx 0.53$ for a hierarchic mass spectrum $M_{\phi_{I}} \ll M_{\Theta} \ll M_{G^{\prime}}$. Mediated by an off-shell $G^{\prime}$, the three-body decay channel $\Theta \rightarrow q \bar{q} \phi_{I}$ has the decay width as

$$
\Gamma\left(\Theta \rightarrow j j \phi_{I}\right) \approx 5 \Gamma\left(\Theta \rightarrow \bar{t} t \phi_{I}\right)=\frac{5 \alpha_{s}^{2} M_{\Theta}^{5}}{576 \pi M_{G^{\prime}}^{4}}\left(1+\tan ^{2} \theta\right)^{2} \mathcal{F}\left(M_{\Theta}^{2} / M_{G^{\prime}}^{2}, M_{\phi_{I}}^{2} / M_{G^{\prime}}^{2}\right)
$$

with the function $\mathcal{F}(x, y)$ defined in ref. [2] and having the limited value $\mathcal{F}(x, y) \stackrel{y \ll x \ll 1}{\longrightarrow} 1$. In the limit of $M_{\phi_{I}} \ll M_{\Theta} \ll M_{G^{\prime}}$, the ratio of the two decay channels is approximately

$$
\frac{\Gamma\left(\Theta \rightarrow j j \phi_{I}\right)}{\Gamma(\Theta \rightarrow g g)} \approx 0.18 \times\left(\frac{\tan \theta}{0.3}\right)^{2}\left(\frac{M_{\Theta} / M_{G^{\prime}}}{1 / 3}\right)^{2} .
$$


So, for a small value of $\tan \theta$ and $M_{\Theta} / M_{G^{\prime}}$, the dominant decay channel is mainly $\Theta \rightarrow g g$. We will not consider other small decay channels like $\Theta \rightarrow q q \bar{q} \bar{q}$ through two off-shell $G^{\prime}$ 's, which is even more suppressed.

The decay modes of $\phi_{I}$ are much more complicated due to its $\mathcal{P}$-even and $\mathcal{C}$-odd nature. For a heavier $\phi_{I}$ above around $500 \mathrm{GeV}$, the main decay channel is one-loop four-body into $g g q \bar{q}$ with the decay width as

$$
\Gamma\left(\phi_{I} \rightarrow q \bar{q} g g\right)=\frac{\alpha_{s}^{5}}{840(12 \pi)^{4}}\left(C_{\Theta}+C_{\Theta}^{\prime}\right)^{2} \frac{\left(1+\tan ^{2} \theta\right)^{4}}{\tan ^{2} \theta} \frac{M_{\phi_{I}}^{11}}{M_{\Theta}^{4} M_{G^{\prime}}^{6}},
$$

with $C_{\Theta} \approx 4.6$ for $M_{\phi_{I}} \ll M_{\Theta} \ll M_{G^{\prime}}$ and $C_{\Theta}^{\prime}$ expected to be $\mathcal{O}(1)$. For a lighter $\phi_{I}$, other two-body three-loop decay channels $\phi_{I} \rightarrow W^{+} W^{-}, Z Z, Z \gamma$ could be dominant, although the lifetime of $\phi_{I}$ could be long enough to have displaced vertexes at the LHC. Given the uncertain results from the three-loop calculations, we will simply assume that the decay branching fraction of $\phi_{I} \rightarrow q \bar{q} g g$ is close to $100 \%$ in our following analysis. We will also ignore the possibly long lifetime of $\phi_{I}$ and simply assume that $\phi_{I}$ decays promptly inside detectors. The long-lived $\phi_{I}$ case requires its own dedicated studies as in refs. [22, 23].

\section{Productions and new signatures at the LHC}

At the LHC and for a heavy coloron above around $3 \mathrm{TeV}$, the main production is a single resonance production. Using the narrow-width approximation and multiplying the cross section by the $K$-factor of 1.2 [24], the $G^{\prime}$ production cross section is approximately $(20,1.8,0.20,0.023) \mathrm{pb}$ for $M_{G^{\prime}}=2,3,4,5 \mathrm{TeV}$ for $\tan \theta=1.0$, using the MSTW parton distribution function [25].

For the three main decay channels in (2.2), the simplest signature to search for the coloron is the traditional one of a dijet resonance. Indeed, both ATLAS and CMS collaborations have set upper constraints on the production cross section times the dijet branching fraction $[18,19]$. Using the signal acceptance of around $0.6[18]$, we will recast their constraints on coloron model parameter space based on the $36 \mathrm{fb}^{-1}$ data. We will also make a simple projection of their sensitivity at $100 \mathrm{fb}^{-1}$. For a small value of $\tan \theta$ around 0.2 , the branching fraction into dijet can be as small as $5 \%$, so a weaker constraint on the coloron mass, $M_{G^{\prime}} \gtrsim 2 \mathrm{TeV}$.

The next type of signature is $p p \rightarrow G^{\prime} \rightarrow(\Theta \rightarrow g g)(\Theta \rightarrow g g)$, with a pair of dijet resonances that form a four-jet resonance. Depending on the $\Theta$ particle mass, its pairproduction from QCD interactions may also be important. The possible three-body decay channel, $\Theta \rightarrow \phi_{I} q \bar{q}$, can generate additional signatures with a larger multiplicity of jets. On other hand, due to the small branching fraction, at least for the benchmark spectrum considered later, we don't consider the three-body decays of $\Theta$ in this paper. There are no dedicated experimental searches for a four-jet resonance with subsequent dijet resonances at the LHC yet, and the searching result from CDF Collaboration [43] on this decay mode is much lighter than the mass range we are considering. For the searches for a pair of dijet resonances without a four-jet resonance [26, 27], we have checked and found that they do not constrain the ReCoM parameter space considered later. 


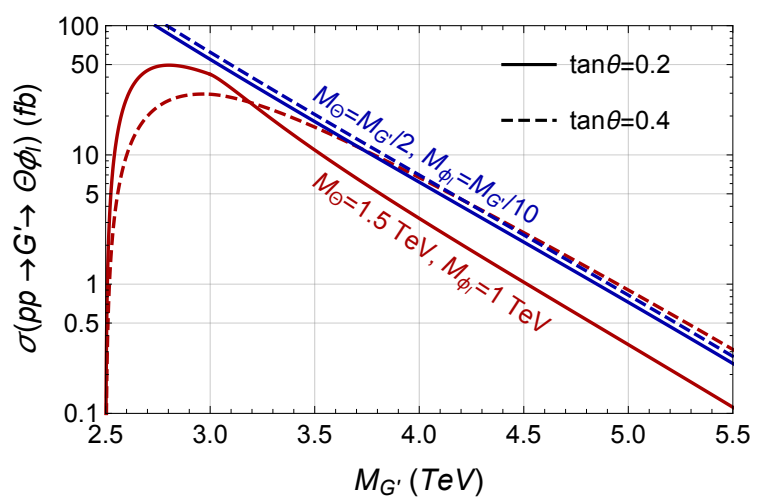

Figure 3. Production cross section times branching ratio of $p p \rightarrow G^{\prime} \rightarrow \Theta \phi_{I}$ at the $13 \mathrm{TeV}$ LHC.

The third type of signature has the process of $p p \rightarrow G^{\prime} \rightarrow(\Theta \rightarrow g g)\left(\phi_{I} \rightarrow q \bar{q} g g\right)$, which will be the focus of this paper. For a heavier $\phi_{I}$ above around $1 \mathrm{TeV}$, the four partons from its decay are likely to form four jets, so the coloron $G^{\prime}$ behaves as a six-jet resonance. The schematic signature at the LHC is shown in the left panel of figure 1. On the other hand, if $\phi_{I}$ is significantly lighter than the mass difference of $G^{\prime}$ and $\Theta$ or $M_{\phi_{I}} \ll M_{G^{\prime}}-M_{\Theta}$, the $\phi_{I}$ from the coloron decay could be boosted such that the four partons from its decay may be grouped into a single fat jet. For this case, we will have the signature of $p p \rightarrow G^{\prime} \rightarrow(\Theta \rightarrow g g) J_{\phi_{I}}$, with $J_{\phi_{I}}$ as a four-prong fat jet. The corresponding schematic signature at the LHC is shown in the right panel of figure 1.

To develop detailed search strategies to search for a coloron as a multi-jet resonance, we introduce two benchmark spectra. For the first one, we fix $M_{\Theta}=1.5 \mathrm{TeV}$ and $M_{\phi_{I}}=1 \mathrm{TeV}$, which represents the left panel of figure 1. For the second one, we choose a hierarchic spectrum with $M_{\Theta} / M_{G^{\prime}}=1 / 2$ and $M_{\phi_{I}} / M_{G^{\prime}}=1 / 10$, for which the $\phi_{I}$ is likely boosted and matches to the situation in the right panel of figure 1 . At the $13 \mathrm{TeV} \mathrm{LHC}$, we show the corresponding production cross section times branching ratio of $p p \rightarrow G^{\prime} \rightarrow \Theta \phi_{I}$ for different coloron masses in figure 3. Fixing $M_{G^{\prime}}=4 \mathrm{TeV}$ and $\tan \theta=0.25$, we show the model information for the two benchmark points (BP's) in table 1.

\section{Search strategies and estimated sensitivities}

In this section, we will develop two independent search strategies for the two types of signatures shown in figure 1. For both searches, we will try to reconstruct the coloron resonances. For the second case, we will also try to reconstruct the invariant mass of $\Theta$ and the jet mass associated with the $J_{\phi_{I}}$ fat jet. Before we dive into the individual cases, we first briefly mention our simulation procedures.

The dominant SM background for the signature we are searching for is the QCD multijet production. The other backgrounds including weak gauge bosons plus jets and top quarks are shown to be subdominant [28]. To simulate the background we generate the process $p p \rightarrow j j+j j j+j j j j$ at parton level with MadGraph 5 [29]. The parton shower, particle decay, and hadronization processes are handled by PYTHIA 6 [30]. The 


\begin{tabular}{|c|c|c|}
\hline & BP1 & BP2 \\
\hline $\begin{array}{c}\left(M_{G^{\prime}}, M_{\Theta}, M_{\phi_{I}}\right) \\
\tan \theta\end{array}$ & $\begin{array}{c}(4,1.5,1) \mathrm{TeV} \\
0.25\end{array}$ & $\begin{array}{c}(4,2,0.4) \mathrm{TeV} \\
0.25\end{array}$ \\
\hline$B\left(G^{\prime} \rightarrow \Theta \Theta\right) \quad B\left(G^{\prime} \rightarrow \Theta \phi_{I}\right)$ & $41.5 \% \quad 38.1 \%$ & $0 \quad 61.3 \%$ \\
\hline$B\left(G^{\prime} \rightarrow j j\right) \quad B\left(G^{\prime} \rightarrow t \bar{t}\right)$ & $17.0 \% \quad 3.4 \%$ & $32.3 \% \quad 6.4 \%$ \\
\hline$\Gamma_{\text {tot }}\left(G^{\prime}\right)$ & $98 \mathrm{GeV}$ & $52 \mathrm{GeV}$ \\
\hline$B(\Theta \rightarrow g g)$ & $99.1 \%$ & $86.5 \%$ \\
\hline$B\left(\Theta \rightarrow q \bar{q} \phi_{I}\right)$ & $0.9 \%$ & $13.5 \%$ \\
\hline$B(\phi \rightarrow q \bar{q} g g)$ & $100 \%$ & $100 \%$ \\
\hline$\sigma\left(p p \rightarrow G^{\prime} \rightarrow \Theta \Theta\right)$ & $5.1 \mathrm{fb}$ & $0 \mathrm{fb}$ \\
\hline$\sigma\left(p p \rightarrow G^{\prime} \rightarrow \Theta \phi_{I}\right)$ & $4.7 \mathrm{fb}$ & $7.5 \mathrm{fb}$ \\
\hline
\end{tabular}

Table 1. Two model benchmark points with some properties of particle decay branching fractions and production cross sections at the $13 \mathrm{TeV}$ LHC.

MLM scheme [31] is employed to handle the matching between matrix element and parton shower calculations. Delphes 3 [32] is utilized to carry out a fast detection simulation with the CMS setup. Jets are clustered using the anti- $k_{\mathrm{T}}$ algorithm [33] with a radius parameter of $R=0.5$, and are accepted only when they have $|\eta|<2.5$ and $p_{T}>50 \mathrm{GeV}$. We also impose a multi-jet trigger requirement with $H_{T}>800 \mathrm{GeV}$ with $H_{T}$ defined as a scalar summation of all jet $p_{T}$ 's [34]. To justify our background simulation, we have also checked the corresponding kinematic distributions against the multi-jet searches for microscopic black holes $[28,35]$ and found good agreement. The multi-jet search results have also been applied to our model and do not constrain the model parameter space.

For the signal events and other than using FeynRules [36] to implement the ReCoM model, the other Monte Carlo simulation tools are largely the same as background simulation. The only difference is that we use PYTHIA 8 [37] instead of PYTHIA 6 such that the four-body decay of $\phi_{I}$ can be handled. Even though that we will concentrate on the $p p \rightarrow G^{\prime} \rightarrow \Theta \phi_{I}$ channel to generate six partons in the final state, we also include the possible signal contaminations of $p p \rightarrow \Theta \Theta$ from both $G^{\prime}$-mediated and QCD interactions.

\subsection{Six-jet resonance search for coloron}

For the signatures in the left panel of figure 1, the coloron decays into six partons, which could become six isolated jets after parton shower, hadronization and ordinary jet reconstructions. Given the possibility of initial and final state radiations, our general search strategy is very simple: a "bump" search of resonances composed of six or more energetic jets. One may also hope to construct the intermediate $\Theta$ and $\phi_{I}$ resonances to further reduce the QCD backgrounds. Given the large combinatorial of 15, the reconstructions of $\Theta$ and $\phi_{I}$ are not efficient and will not be considered here.

There are three useful variables to increase the signal significance: the number of jets $N_{j},|\eta|$ for jet selection and the invariant mass $M_{\text {inv }}$ of all jets. For the first variable $N_{j}$, we show the normalized distributions of jet multiplicities for both QCD background and the 

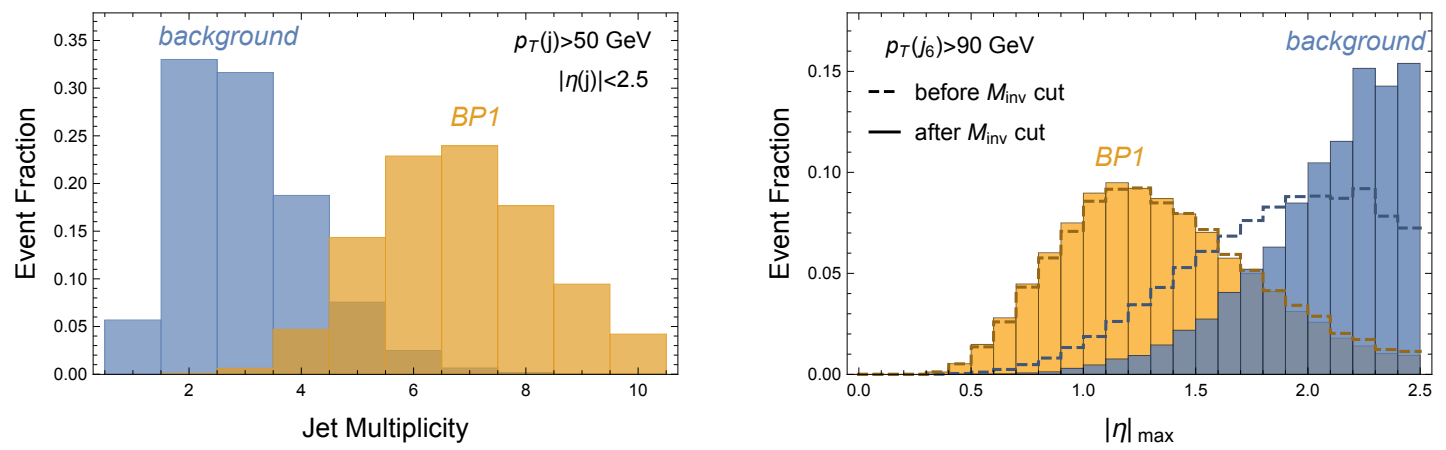

Figure 4. Left panel: normalized jet-multiplicity distribution for the QCD background and the benchmark model point BP1. Right panel: normalized distributions for $|\eta|_{\max }$ (the maximum $|\eta|$ for leading six jets) with and without the cuts on the total invariant mass $\left|M_{\mathrm{inv}}-M_{G^{\prime}}\right| / M_{G^{\prime}}<15 \%$.

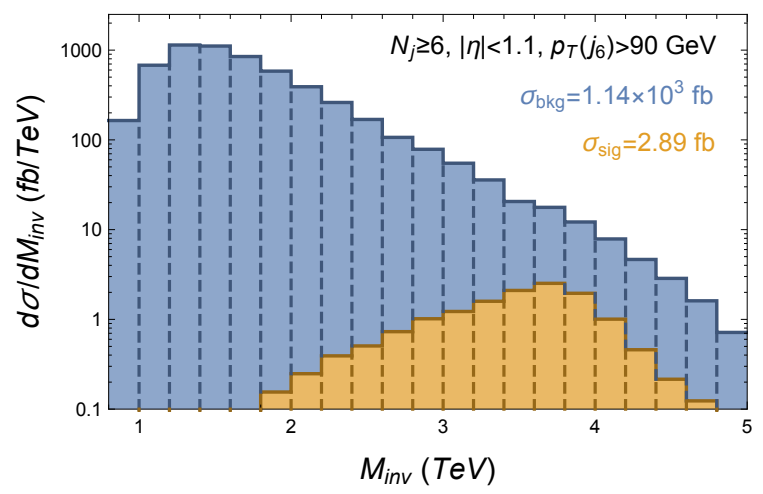

Figure 5. The signal and background invariant mass distributions for the number of jets equal to or above six. The cuts on other kinematic variables in (4.1) have also been imposed. For this BP1 signal, $M_{G^{\prime}}=4 \mathrm{TeV}$.

signal BP1 in the left panel of figure 4. It is clear that the QCD background has the jet multiplicity distribution peaked at two or three, while the BP1 signal events have a peak at around six and seven. So, imposing a cut on the jet multiplicity with $N_{j} \geq 6$ can reduce the background and increase the BP1 signal sensitivity.

Similar to the searches for quark compositeness and because of the Rutherforld scattering in QCD, requiring the jets to be more central or with a small value of $|\eta|$ can improve the signal sensitivity $[19,38]$. In the right panel of figure 4 , we show the variable $|\eta|_{\max }$ (the maximum value of $|\eta|$ 's of the leading six jets) distributions for both signal and background with or without the additional invariant mass cut $\left|M_{\text {inv }}-M_{G^{\prime}}\right| / M_{G^{\prime}}<15 \%$. It is clear that after imposing the invariant mass cut, the QCD background has a small fraction of events with $|\eta|_{\max }$ below around 1.0. So, requiring an upper bound on $|\eta|$ for all jets can efficiently increase the discovery sensitivity.

For the invariant mass distributions, we show both the background and the signal differential cross section distributions in figure 5, after imposing the optimized cuts on other variables. To take into account of the final state radiation jets, the invariant mass 


\begin{tabular}{|c|c|ccc|ccc|}
\hline Cut-flow & Background & \multicolumn{3}{|c|}{ BP1 } & \multicolumn{3}{|c|}{ BP2 } \\
& $\sigma(\mathrm{fb})$ & $\sigma(\mathrm{fb})$ & $\epsilon$ & $S / \sqrt{B}$ & $\sigma(\mathrm{fb})$ & $\epsilon$ & $S / \sqrt{B}$ \\
\hline$N_{j} \geqslant 6,|\eta|<2.5$ & $1.37 \times 10^{5}$ & 8.72 & $80.6 \%$ & 0.24 & 3.81 & $50.5 \%$ & 0.10 \\
$N_{j} \geqslant 6,|\eta|<1.1$ & $1.02 \times 10^{4}$ & 5.09 & $47.1 \%$ & 0.50 & 1.74 & $23.0 \%$ & 0.17 \\
$p_{T}\left(j_{6}\right)>90 \mathrm{GeV}$ & $1.14 \times 10^{3}$ & 2.89 & $26.7 \%$ & 0.86 & 0.75 & $10.0 \%$ & 0.22 \\
$\left|M_{\text {inv }}-M_{G^{\prime}}\right| / M_{G^{\prime}}<15 \%$ & 13.14 & 1.62 & $15.0 \%$ & 4.5 & 0.47 & $6.2 \%$ & 1.3 \\
\hline
\end{tabular}

Table 2. Cut flow of signal and background cross sections and acceptances. Also shown are the signal significances with an integrated luminosity of $100 \mathrm{fb}^{-1}$.

\begin{tabular}{|c|c|c|c|c|c|}
\hline \multicolumn{2}{|c|}{$p p \rightarrow G^{\prime} \rightarrow \Theta \phi_{I}$} & \multicolumn{2}{|c|}{$p p \rightarrow G^{\prime} \rightarrow \Theta \Theta$} & \multicolumn{2}{c|}{$p p \rightarrow \Theta \Theta(\mathrm{QCD})$} \\
\hline$\sigma(\mathrm{fb})$ & $\epsilon$ & $\sigma(\mathrm{fb})$ & $\epsilon$ & $\sigma(\mathrm{fb})$ & $\epsilon$ \\
\hline 0.90 & $19.3 \%$ & 0.66 & $12.9 \%$ & 0.07 & $6.2 \%$ \\
\hline
\end{tabular}

Table 3. Contributions to the signal strength of the BP1 from different subprocesses.

variable, $M_{\text {inv }}$, includes all jets with $p_{T}>50 \mathrm{GeV}$. For the signal distributions, one can see that the reconstructed distribution is peaked at around but slightly below the coloron mass, which is due to both energy resolutions and missing jets in the more forward directions. Due to the detector energy resolution, the width of the peak is around $15 \%$ of the coloron mass, so we will choose the mass window cut to be $\left|M_{\text {inv }}-M_{G^{\prime}}\right| / M_{G^{\prime}}<15 \%$. For the background distribution, there is a peak at around $1.4 \mathrm{TeV}$, which is due to the trigger requirement of $H_{T}>800 \mathrm{GeV}$ and a simulation cut of requiring the invariant mass of partons above $1 \mathrm{TeV}$.

All together, we have the following optimized cuts for the BP1

$$
N_{j} \geqslant 6, \quad|\eta|<1.1, \quad p_{T}\left(j_{6}\right)>90 \mathrm{GeV}, \quad\left|M_{\mathrm{inv}}-M_{G^{\prime}}\right| / M_{G^{\prime}}<15 \% .
$$

To demonstrate the efficiencies of different cuts, we show the cut flow of the background and signal events in table 2. After imposing all the cuts in (4.1), the signal significance for the BP1 has $S / \sqrt{B} \approx 4.5$ at the $13 \mathrm{TeV}$ LHC with $100 \mathrm{fb}^{-1}$, which definitely shows high discovery potential at the LHC. In table 3 , we show the contributions to signal strength from different subprocesses of the BP1. A simple anatomy of the two subprocesses is displayed in figure 6 , where the distributions of jet multiplicity and $p_{T}\left(j_{6}\right)$ are shown. The $p p \rightarrow \Theta \Theta$ process has a smaller jet multiplicity than $\Theta \phi_{I}$ process, and it also has a softer $p_{T}\left(j_{6}\right)$. One can see that even though our search strategy for the six-jet resonance is designed for the $\Theta \phi_{I}$ signal, it can also cover the signal of $p p \rightarrow \Theta \Theta$ with $\Theta$ mainly decaying into two gluons together with additional final-state radiations.

In table 2, we also show the acceptances and the signal significance for the BP2. Compared to the BP1, the signal acceptance is smaller by a factor of more than two. So, the search strategy developed in this section may not be an optimized one for the BP2. A simple reason for this is that the $\phi_{I}$ boson is boosted such that it only appears as one high $p_{T}$ jet. The requirement of having at least six jets with $p_{T}$ above $90 \mathrm{GeV}$ reduces the BP2 

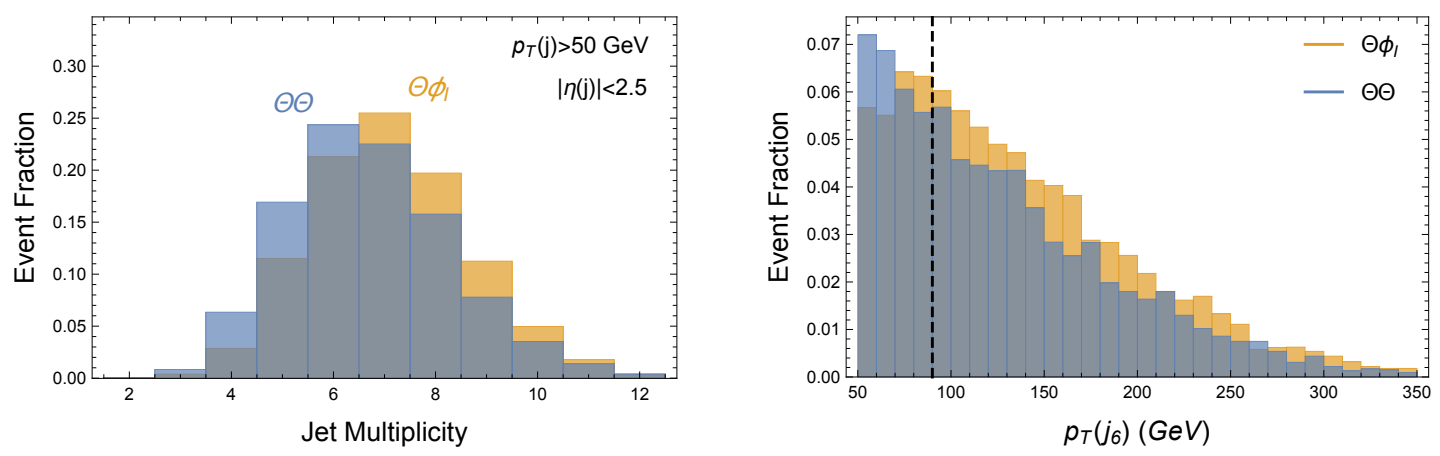

Figure 6. Left panel: normalized jet-multiplicity distribution for the two subprocesses $p p \rightarrow \Theta \phi_{I}$ and $p p \rightarrow \Theta \Theta$ in the benchmark model point BP1. Right panel: normalized distributions for $p_{T}\left(j_{6}\right)$. The black dashed line indicates the $p_{T}\left(j_{6}\right)>90 \mathrm{GeV}$ cut we use in our analysis.

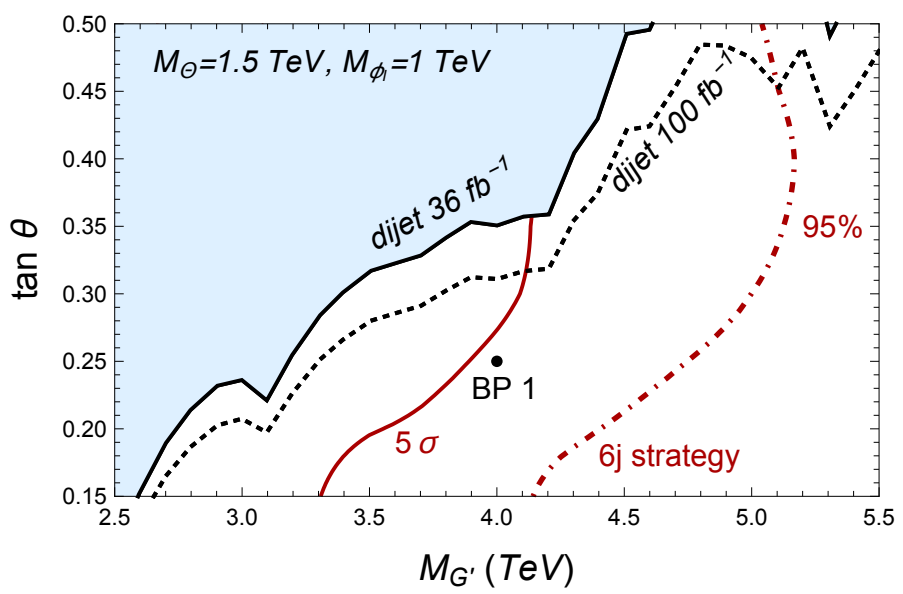

Figure 7. Estimated sensitivity for different $M_{G^{\prime}}$ and $\tan \theta$ and fixed $M_{\Theta}=1.5 \mathrm{TeV}$ and $M_{\phi_{I}}=1 \mathrm{TeV}$ at the $13 \mathrm{TeV}$ with $100 \mathrm{fb}^{-1}$. The current and projected $95 \% \mathrm{CL}$ constraints from the dijet resonance searches are also shown in the black solid and dotted lines [18].

signal strength. In the section 4.2 , we will develop a better fat-jet-based search strategy for the BP2.

Fixing $M_{\Theta}=1.5 \mathrm{TeV}$ and $M_{\phi_{I}}=1 \mathrm{TeV}$, we scan the parameter space of $M_{G^{\prime}}$ and $\tan \theta$ with a step width of $0.25 \mathrm{TeV}$ and 0.05 respectively. At each grid point, we impose the cuts in eq. (4.1) for the corresponding $M_{G^{\prime}}$ to select signal and background and calculate the significance. The estimated $5 \sigma$ discovery and 95\% CL exclusion limits are given in figure 7 . Also shown in this figure are the $95 \%$ CL constraints from dijet resonance searches [18] with $36 \mathrm{fb}^{-1}$ luminosity and the simple projected sensitivity at $100 \mathrm{fb}^{-1}$. A signal acceptance factor of 0.6 is used to obtain the limits in the black solid and dashed lines. Comparing the dijet and six-jet resonance sensitivities, it is clear from this figure that a wide range of parameter space of ReCoM may have $5 \sigma$ discovery potential based on searches for a six-jet resonance. Due to the limitation of our Monte Carlo tools and the uncertainties on the tails of the background kinematic distributions, the calculated significance should have some uncertainties. However, we want to stress that our search strategy is a bump search. 

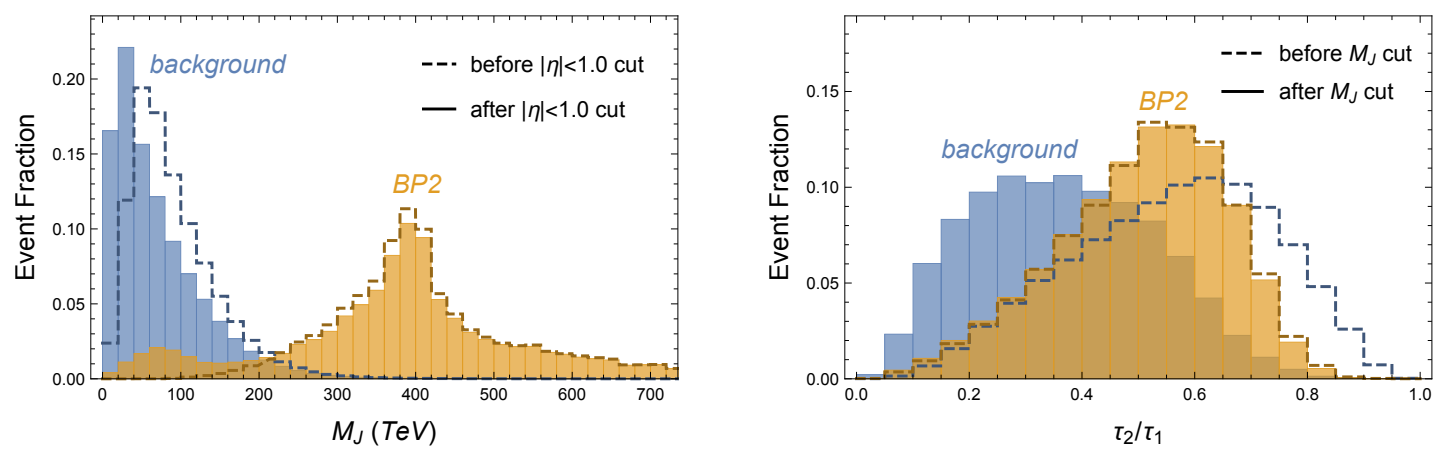

Figure 8. Left panel: the normalized leading fat-jet mass distributions for the benchmark model point BP2 and QCD background with or without the $|\eta|<1.0$ cut. The anti- $k_{T}$ jet-finding algorithm with $R=1.0$ has been used. Right panel: normalized $\tau_{2} / \tau_{1}$ distributions of the most massive jet for the signal and background with or without the fat-jet mass cut in (4.2).

The real experimental searches can use a data-driven way to hunt for the new resonance and may not rely on Monte Carlo simulations.

\subsection{Coloron as a resonance containing a fat jet $J_{\phi_{I}}$}

For the second type of spectra with $M_{\phi_{I}} \ll M_{G^{\prime}}-M_{\Theta}$, the four partons from $\phi_{I}$ decays are likely to be collimated and form a single fat-jet $J_{\phi_{I}}$ (see the right panel of figure 1 for the schematic illustration of this type of signals at the LHC). Meanwhile, the two gluons from $\Theta$ decays will behave as ordinary jets. Therefore, we implement a two-step jet reconstruction method to analyze the events in order to reveal the above features. For all the proto-jets from parton shower, we first use the anti- $k_{T}$ jet-finding algorithm [33] with $R=1.0$ to identify the possible $J_{\phi_{I}}$. More specifically, we choose the $R=1.0$ jet with the largest jet mass $M_{J}$ as $J_{\phi_{I}}$. We then remove the components inside $J_{\phi_{I}}$ from the list and re-cluster the remaining proto-jets into ordinary jets using the $R=0.5$ anti- $k_{T}$ algorithm. Both large- $R$ jets and small- $R$ jets are required to have $|\eta|<2.5$.

To optimize the sensitivity for the second type of spectra with a fat $\phi_{I}$ jet, we have found four useful variables, the fat jet mass $M_{J}$, an N-subjettiness variable $\tau_{2} / \tau_{1}$ [39], the invariant mass of non-fat jet $M_{\mathrm{inv}}(j) \equiv M\left[\sum p_{\mu}(j)\right]$ (all ordinary jets are included) and the total invariant mass of the fat jet and ordinary jets $M_{\text {inv }}(J, j) \equiv M\left[p_{\mu}(J)+\sum p_{\mu}(j)\right]$. All of those variables except $\tau_{2} / \tau_{1}$ are intuitively understood. Naively, since there are four partons from $\phi_{I}$ decays, one may wonder about another variable $\tau_{4} / \tau_{3}$, which is more suitable for a four-prong jet. The decay of $\phi_{I} \rightarrow g g q \bar{q}$, however, is through off-shell particles rather than on-shell intermediate particles. As a result, the parton energies are more hierarchical, which makes $\tau_{4} / \tau_{3}$ less useful to suppress QCD background (see appendix A for detail).

In the left panel of figure 8, we show the normalized fat jet mass distributions for both BP2 and the QCD background. Among more than one possible fat jets, we take the one with the largest jet mass. It is clear from this plot that imposing a cut on the fat-jet mass above around $300 \mathrm{GeV}$ can effectively increase $S / \sqrt{B}$ for the BP2. For the signal events, sometimes the truth $J_{\phi_{I}}$ may not pass the $|\eta|<1.0$ cut, so a non- $\phi_{I}$ jet is chosen as the leading fat-jet candidate, which may have a smaller jet mass and show up in the left part 

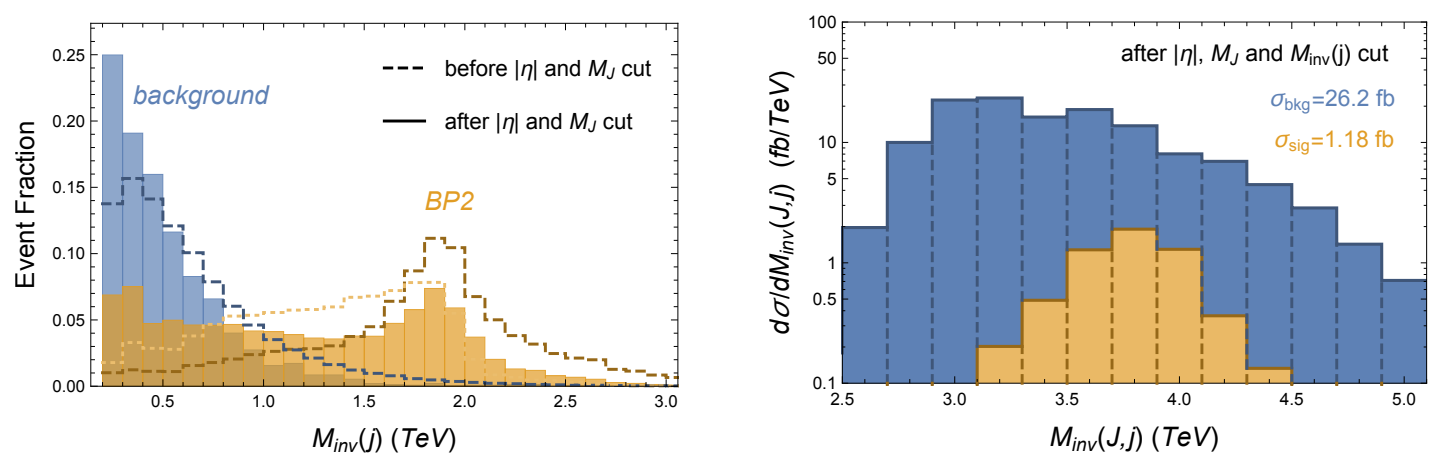

Figure 9. Left panel: normalized distributions of the invariant mass $M_{\mathrm{inv}}(j)$ of all remaining normal jets for the BP2 signal and background before or after the $|\eta|$ and $M_{J}$ cuts. The yellow dotted line shows the signal distribution if one only includes two leading $p_{T}$ ordinary jets. Right panel: the invariant mass $M_{\mathrm{inv}}(J, j)$ distributions for both the QCD background and the BP2 signal.

of the signal distribution. In the right panel of figure 8 , we show the normalized $\tau_{2} / \tau_{1}$ distributions for the signal and background. Before we impose the $M_{J}>0.8 M_{\phi_{I}}$ cut, the QCD background is peaked at higher values of $\tau_{2} / \tau_{1}$ than the signal. After the $M_{J}$ cut, the QCD background has a peak at lower values of $\tau_{2} / \tau_{1}$, which means that the two-prong feature of the QCD background becomes even more dramatic than the signal. Imposing a lower-limit cut on $\tau_{2} / \tau_{1}$ can therefore increase $S / \sqrt{B}$.

For the invariant mass $M_{\mathrm{inv}}(j)$ of ordinary jets excluding the fat-jet, we show the normalized distributions in the left panel of figure 9 . As one can see, before the $|\eta|$ and $M_{J}$ cuts, the signal distribution has a nice peak around the $\Theta$ mass. After those two cuts, the spectrum is distorted with a long tail in the small invariant mass region. This is because some jets from $\Theta$ decay may be mis-identified as the fat-jet if the actual $J_{\phi_{I}}$ does not pass the $|\eta|$ or $M_{J}$ cuts. For this variable, $M_{\text {inv }}(j)$, we have included all ordinary jets passing the basic cuts. In the yellow dotted line, we also show the signal distribution if one only includes two leading $p_{T}$ ordinary jets and before the $|\eta|$ and $M_{J}$ cuts. Although it also has a peak at around $M_{\Theta}$, it does not have a nice bump structure as the yellow dashed line and has a long-tail in lower values due to the final state radiation effect. In the right panel of figure 9 , we show the invariant mass $M_{\text {inv }}(J, j)$ distributions after imposing the $|\eta|$, $M_{J}$ and $M_{\text {inv }}(j)$ cuts. For the signal events, they have a peak located at around the true coloron mass of $4 \mathrm{TeV}$. For the QCD background events, they show a decreasing behavior after around $3 \mathrm{TeV}$, while the peak at around $3 \mathrm{TeV}$ is mainly due to the cut of requiring $M_{\text {inv }}(j)$ close to the $\Theta$ mass of $2 \mathrm{TeV}$ for this BP2.

Using the BP2 as an example, we optimize the cuts for the few kinematic variables in this section together with the $\eta$ cut as

$$
\begin{array}{lll}
|\eta|<1.0, & \tau_{2} / \tau_{1}>0.5, & M_{J}>0.8 M_{\phi_{I}}, \\
\left|M_{\mathrm{inv}}(j)-M_{\Theta}\right| / M_{\Theta}<15 \%, & \left|M_{\mathrm{inv}}(J, j)-M_{G^{\prime}}\right| / M_{G^{\prime}}<15 \% .
\end{array}
$$

For the $p_{T}$ cut used in (4.1) of the previous section, it is not efficient to increase the signal significance for the $\mathrm{BP} 2$, which is due to the cuts on the variables $M_{J}$ and $M_{\mathrm{inv}}(j)$. So, we 


\begin{tabular}{|c|c|ccc|}
\hline Cut-flow & $\begin{array}{c}\text { Background } \\
\sigma(\mathrm{fb})\end{array}$ & $\sigma(\mathrm{fb})$ & $\epsilon$ & $S / \sqrt{B}$ \\
\hline$M_{J}>0.8 M_{\phi_{I}},|\eta|<2.5$ & $1.60 \times 10^{4}$ & 6.08 & $81.1 \%$ & 0.48 \\
$M_{J}>0.8 M_{\phi_{I}},|\eta|<1.0$ & $1.13 \times 10^{4}$ & 5.43 & $72.5 \%$ & 0.51 \\
$\left|M_{\text {inv }}(j)-M_{\Theta}\right| / M_{\Theta}<15 \%$ & 26.6 & 1.18 & $15.7 \%$ & 2.28 \\
$\left|M_{\text {inv }}(J, j)-M_{G^{\prime}}\right| / M_{G^{\prime}}<15 \%$ & 12.1 & 1.06 & $14.2 \%$ & 3.06 \\
$\tau_{2} / \tau_{1}>0.5$ & 2.93 & 0.61 & $8.2 \%$ & 3.59 \\
\hline
\end{tabular}

Table 4. Cut flow of signal and background cross sections and acceptances, based on the fat-jet analysis. Also shown are the signal significances with an integrated luminosity of $100 \mathrm{fb}^{-1}$.

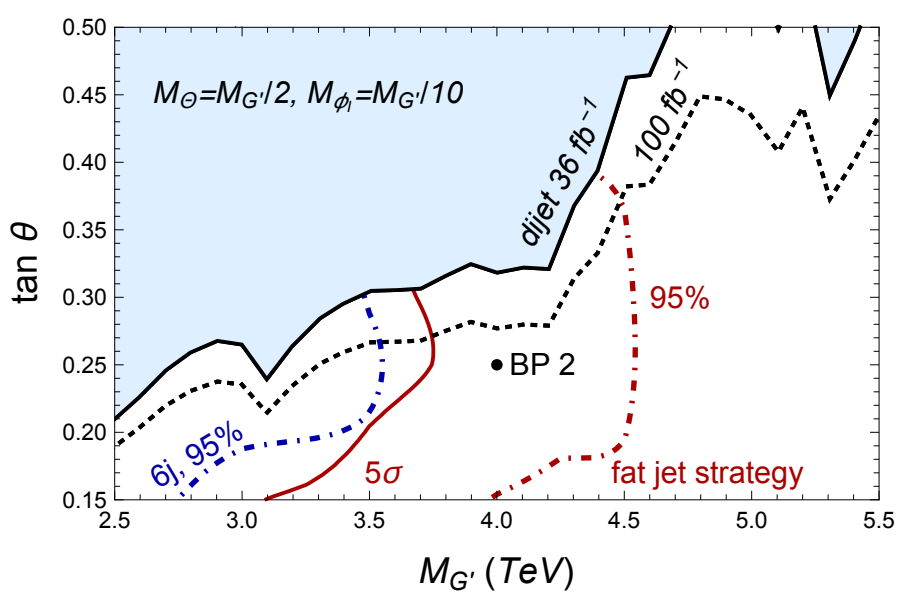

Figure 10. The same as figure 7 , but based on the fat-jet analysis and fixing the ratios of $M_{\Theta} / M_{G^{\prime}}=1 / 2$ and $M_{\phi_{I}} / M_{G^{\prime}}=1 / 10$. The sensitivities for the signal are based on the $13 \mathrm{TeV}$ LHC with $100 \mathrm{fb}^{-1}$. As a comparison, we also show the sensitivities based on the six-jet resonance search strategy in the blue dot-dashed line.

just keep the basic cuts of $p_{T}(j)>50 \mathrm{GeV}$. We also show the cut-flow for the signal BP2 in table 4 .

Using the cuts from (4.2), we scan the parameter space in $\tan \theta$ and $M_{G^{\prime}}$ for the fixed ratios of $M_{\Theta} / M_{G^{\prime}}=1 / 2$ and $M_{\phi_{I}} / M_{G^{\prime}}=1 / 10$ and show the $5 \sigma$ discovery and $95 \%$ CL exclusion limits in figure 10. In the blue dot-dashed line, we also show the $95 \%$ CL exclusion limit based on the six-jet resonance search strategy in section 4.1. The comparison between the two sets of exclusion lines shows that the fat-jet based method can probe a larger region of model parameter space. For a wide range of mixing angles, the LHC can discover a coloron via the multi-jet channel up to a mass of around $3.5 \mathrm{TeV}$ and exclude the existence of a coloron at 95\% CL up to a mass of around $4.5 \mathrm{TeV}$.

\section{$5 \quad$ Discussion and conclusions}

In this paper, we have developed two search strategies based on the predicted signatures in the ReCoM. Both strategies could be applied to other models with similar signatures. 
For instance, one could have the process of $p p \rightarrow Z^{\prime} \rightarrow t^{\prime} \bar{t}^{\prime} \rightarrow W^{+} b W^{-} \bar{b} \rightarrow 4 j 2 b$ with a $Z^{\prime}$ decaying into some top-partner $t^{\prime}$. The $Z^{\prime}$ behaves as a six-jet resonance with the hadronic $W$ 's as possible fat-jets. Although there are non-resonance searches based on multi-jet final states $[28,35,41]$ at the LHC, looking for additional "bumps" that are composed a large number of jets has a great discovery potential.

We have concentrated on the prompt decays of new particles in this paper. As pointed out in ref. [2], the color-singlet scalar $\phi_{I}$ may have a long decay lifetime if its mass is below around $500 \mathrm{GeV}$, with the main decay channel as two electroweak gauge bosons. It is feasible that the discovery potential is even higher than both results in figure 7 and 10, if the displaced vertex can be identified inside the detector. In the ReCoM and because of a U(1) symmetry, the $\phi_{I}$ could be a long-lived PNGB. Although its direct couplings to the SM particles are suppressed, it can be produced via decays of other heavy new particles like $G^{\prime}$ considered in this paper. A similar situation may happen in other beyond-SM models. For instance, the top-partner $t^{\prime}$ can decay into a top quark plus a long-lived PNGB [42]. Searching for this type of signatures with long-lived particles at the LHC can explore a wide range of new physics models.

In summary, we have performed a detailed collider study for one type of coloron signatures at the LHC. The coloron can behave as a six-jet resonance from its cascade decays of $G^{\prime} \rightarrow(\Theta \rightarrow g g)\left(\phi_{I} \rightarrow q \bar{q} g g\right)$. For the case with a non-boosted $\phi_{I}$, we have found that performing a bump search for a six-jet resonance can cover a wide and new region of coloron parameter space. For the mixing angle below 0.35 and above the perturbative value of 0.15 , a coloron with a mass from $3.3 \mathrm{TeV}$ to $4.0 \mathrm{TeV}$ can have a $5 \sigma$ discovery chance, while at $95 \% \mathrm{CL}$ its mass can be constrained up to $5 \mathrm{TeV}$. For the second case with a boosted $\phi_{I}$, a fat-jet-based strategy is developed with the $5 \sigma$ discovery limit up to $3.5 \mathrm{TeV}$ and the $95 \%$ CL constraint limit up to $4.5 \mathrm{TeV}$ for the coloron mass. The large list of new signatures for the coloron in ref. [2] and the specific signatures studied in this paper serve as examples for the great discovery potential of new physics based on future LHC data.

\section{Acknowledgments}

We thank Bogdan A. Dobrescu for discussion. The work of YB and SL is supported by the U.S. Department of Energy under the contract DE-SC0017647. The work of QFX is supported by the China Scholarship Council with Grant No. 201704910714.

\section{A $\tau_{4} / \tau_{3}$ distribution for the fat $\phi_{I}$ jet}

In N-subjettiness method, a fat jet is assumed to be made of $N$ subjets. The $\mathrm{N}$-subjettiness variables $\tau_{N}$ is calculated as [39]

$$
\tau_{N}=\frac{1}{d_{0}} \sum_{k} p_{T, k} \min \left\{\Delta R_{1, k}, \Delta R_{2, k}, \cdots, \Delta R_{N, k}\right\}, \quad N=1,2, \ldots,
$$

where $\min \left\{\Delta R_{1, k}, \Delta R_{2, k}, \cdots, \Delta R_{N, k}\right\}$ means minimizing over all possible directions of the $N$ subjets. $\Delta R_{J, k}=\sqrt{(\Delta \eta)^{2}+(\Delta \phi)^{2}}$ is the angular distance between the candidate subjet 


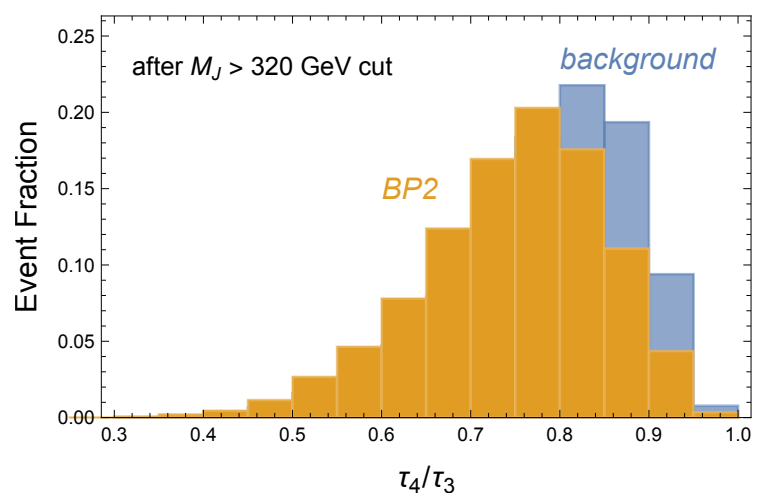

Figure 11. Normalized $\tau_{4} / \tau_{3}$ distributions of the most massive jet for the signal and background after a jet-mass cut.

$J$ and proto-jet $k$, and $d_{0}=\sum_{k} p_{T, k} R_{0}$ with $R_{0}$ the characteristic jet radius used in the jet-finding algorithm. It is believed that the distribution of $\tau_{N} / \tau_{N-1}$ will have a clear distinction from the background if the fat jet is $N$-prong, and this has been well-tested for $W$ and $t$ jets which are 2- and 3-prong [39].

Because of the decay of $\phi_{I} \rightarrow g g q \bar{q}$, a boosted $\phi_{I}$ is anticipated to behave as a fourprong fat-jet. Naively, one should anticipate that the $\mathrm{N}$-subjettiness variable, $\tau_{4} / \tau_{3}$, could be useful to separate signal from background. However, the four-body decays of $\phi_{I}$ is through off-shell $G^{\prime}$ and $\Theta$. As a result, the four partons in the decay products are more hierarchic in energy rather than more democratic, which downgrades its four-prong feature. In figure 11, we show the distributions of $\tau_{4} / \tau_{3}$ for both signal and background, which shows the similarity of the two distributions.

The definition of $\tau_{N}$ can be generalized by raising the power of angular separation $\Delta R_{J, k}$ from 1 to $\beta$ [40]. In our analysis, we have also checked the result for $\beta=2$ and find no significant difference for $\tau_{4} / \tau_{3}$.

Open Access. This article is distributed under the terms of the Creative Commons Attribution License (CC-BY 4.0), which permits any use, distribution and reproduction in any medium, provided the original author(s) and source are credited.

\section{References}

[1] Y. Bai and B.A. Dobrescu, Heavy octets and Tevatron signals with three or four b jets, JHEP 07 (2011) 100 [arXiv: 1012.5814] [INSPIRE].

[2] Y. Bai and B.A. Dobrescu, Collider Tests of the Renormalizable Coloron Model, JHEP 04 (2018) 114 [arXiv: 1802.03005] [INSPIRE].

[3] C.T. Hill, Topcolor: Top quark condensation in a gauge extension of the standard model, Phys. Lett. B 266 (1991) 419 [INSPIRE].

[4] C.T. Hill and S.J. Parke, Top production: Sensitivity to new physics, Phys. Rev. D 49 (1994) 4454 [hep-ph/9312324] [INSPIRE]. 
[5] R.S. Chivukula, A.G. Cohen and E.H. Simmons, New strong interactions at the Tevatron?, Phys. Lett. B 380 (1996) 92 [hep-ph/9603311] [INSPIRE].

[6] E.H. Simmons, Coloron phenomenology, Phys. Rev. D 55 (1997) 1678 [hep-ph/9608269] [INSPIRE].

[7] L.J. Hall and A.E. Nelson, Heavy Gluons and Monojets, Phys. Lett. B 153 (1985) 430 [INSPIRE].

[8] P.H. Frampton and S.L. Glashow, Chiral Color: An Alternative to the Standard Model, Phys. Lett. B 190 (1987) 157 [inSPIRE].

[9] J. Bagger, C. Schmidt and S. King, Axigluon Production in Hadronic Collisions, Phys. Rev. D 37 (1988) 1188 [INSPIRE].

[10] C. Kilic, T. Okui and R. Sundrum, Vectorlike Confinement at the LHC, JHEP 02 (2010) 018 [arXiv:0906.0577] [INSPIRE].

[11] B.A. Dobrescu, K. Kong and R. Mahbubani, Massive color-octet bosons and pairs of resonances at hadron colliders, Phys. Lett. B 670 (2008) 119 [arXiv: 0709.2378] [INSPIRE].

[12] J. Sayre, D.A. Dicus, C. Kao and S. Nandi, Searching for Colorons at the Large Hadron Collider, Phys. Rev. D 84 (2011) 015011 [arXiv:1105.3219] [INSPIRE].

[13] Y. Bai and J. Shelton, Composite Octet Searches with Jet Substructure, JHEP 07 (2012) 067 [arXiv:1107.3563] [INSPIRE].

[14] R.S. Chivukula, A. Farzinnia, J. Ren and E.H. Simmons, Constraints on the Scalar Sector of the Renormalizable Coloron Model, Phys. Rev. D 88 (2013) 075020 [Erratum ibid. D 89 (2014) 059905] [arXiv: 1307.1064] [INSPIRE].

[15] R.S. Chivukula, E.H. Simmons, A. Farzinnia and J. Ren, LHC Constraints on a Higgs boson Partner from an Extended Color Sector, Phys. Rev. D 90 (2014) 015013 [arXiv:1404.6590] [INSPIRE].

[16] Y. Bai and B.A. Dobrescu, Minimal SU(3) $\times$ SU(3) Symmetry Breaking Patterns, Phys. Rev. D 97 (2018) 055024 [arXiv:1710.01456] [INSPIRE].

[17] P. Draper, J. Kozaczuk and J.-H. Yu, Theta in new QCD-like sectors, Phys. Rev. D 98 (2018) 015028 [arXiv: 1803.00015] [INSPIRE].

[18] CMS collaboration, Searches for dijet resonances in pp collisions at $\sqrt{s}=13 \mathrm{TeV}$ using data collected in 2016., CMS-PAS-EXO-16-056.

[19] ATLAS collaboration, Search for new phenomena in dijet events using $37 \mathrm{fb}^{-1}$ of pp collision data collected at $\sqrt{s}=13 \mathrm{TeV}$ with the ATLAS detector, Phys. Rev. D 96 (2017) 052004 [arXiv: 1703.09127] [INSPIRE].

[20] ATLAS collaboration, Search for heavy particles decaying into top-quark pairs using lepton-plus-jets events in proton-proton collisions at $\sqrt{s}=13 \mathrm{TeV}$ with the ATLAS detector, Eur. Phys. J. C 78 (2018) 565 [arXiv: 1804.10823] [INSPIRE].

[21] CMS collaboration, Search for $\mathrm{t} \overline{\mathrm{t}}$ resonances in highly boosted lepton+jets and fully hadronic final states in proton-proton collisions at $\sqrt{s}=13 \mathrm{TeV}$, JHEP 07 (2017) 001 [arXiv:1704.03366] [INSPIRE].

[22] CMS collaboration, Search for long-lived charged particles in proton-proton collisions at $\sqrt{s}=13 \mathrm{TeV}$, Phys. Rev. D 94 (2016) 112004 [arXiv: 1609.08382] [InSPIRE]. 
[23] ATLAS collaboration, Search for long-lived, massive particles in events with displaced vertices and missing transverse momentum in $\sqrt{s}=13 \mathrm{TeV} p p$ collisions with the ATLAS detector, Phys. Rev. D 97 (2018) 052012 [arXiv:1710.04901] [InSPIRE].

[24] R.S. Chivukula, A. Farzinnia, J. Ren and E.H. Simmons, Hadron Collider Production of Massive Color-Octet Vector Bosons at Next-to-Leading Order, Phys. Rev. D 87 (2013) 094011 [arXiv: 1303.1120] [INSPIRE].

[25] A.D. Martin, W.J. Stirling, R.S. Thorne and G. Watt, Parton distributions for the LHC, Eur. Phys. J. C 63 (2009) 189 [arXiv:0901.0002] [inSPIRE].

[26] CMS collaboration, Search for pair-produced resonances decaying to jet pairs in proton-proton collisions at $\sqrt{s}=8 \mathrm{TeV}$, Phys. Lett. B $\mathbf{7 4 7}$ (2015) 98 [arXiv:1412.7706] [INSPIRE].

[27] ATLAS collaboration, A search for pair-produced resonances in four-jet final states at $\sqrt{s}=13 \mathrm{TeV}$ with the ATLAS detector, ATLAS-CONF-2017-025.

[28] CMS collaboration, Search for black holes in high-multiplicity final states in proton-proton collisions at $\sqrt{s}=13 \mathrm{TeV}$, Phys. Lett. B 774 (2017) 279 [arXiv:1705.01403] [INSPIRE].

[29] J. Alwall et al., The automated computation of tree-level and next-to-leading order differential cross sections and their matching to parton shower simulations, JHEP 07 (2014) 079 [arXiv: 1405.0301] [INSPIRE].

[30] T. Sjöstrand, S. Mrenna and P.Z. Skands, PYTHIA 6.4 Physics and Manual, JHEP 05 (2006) 026 [hep-ph/0603175] [INSPIRE].

[31] M.L. Mangano, M. Moretti, F. Piccinini and M. Treccani, Matching matrix elements and shower evolution for top-quark production in hadronic collisions, JHEP 01 (2007) 013 [hep-ph/0611129] [INSPIRE].

[32] DELPHES 3 collaboration, J. de Favereau et al., DELPHES 3, A modular framework for fast simulation of a generic collider experiment, JHEP 02 (2014) 057 [arXiv:1307.6346] [INSPIRE].

[33] M. Cacciari, G.P. Salam and G. Soyez, The anti- $k_{t}$ jet clustering algorithm, JHEP 04 (2008) 063 [arXiv: 0802.1189] [INSPIRE].

[34] CMS collaboration, The CMS trigger system, 2017 JINST 12 P01020 [arXiv:1609.02366] [INSPIRE].

[35] CMS collaboration, Search for black holes and sphalerons in high-multiplicity final states in proton-proton collisions at $\sqrt{s}=13 \mathrm{TeV}$, arXiv:1805.06013 [INSPIRE].

[36] A. Alloul, N.D. Christensen, C. Degrande, C. Duhr and B. Fuks, FeynRules $2.0-A$ complete toolbox for tree-level phenomenology, Comput. Phys. Commun. 185 (2014) 2250 [arXiv:1310.1921] [INSPIRE].

[37] T. Sjöstrand et al., An Introduction to PYTHIA 8.2, Comput. Phys. Commun. 191 (2015) 159 [arXiv: 1410.3012] [INSPIRE].

[38] CMS collaboration, Search for new physics in dijet angular distributions using proton-proton collisions at $\sqrt{s}=13 \mathrm{TeV}$ and constraints on dark matter and other models, arXiv: 1803.08030 [INSPIRE].

[39] J. Thaler and K. Van Tilburg, Identifying Boosted Objects with N-subjettiness, JHEP 03 (2011) 015 [arXiv: 1011.2268] [INSPIRE]. 
[40] J. Thaler and K. Van Tilburg, Maximizing Boosted Top Identification by Minimizing $N$-subjettiness, JHEP 02 (2012) 093 [arXiv: 1108.2701] [INSPIRE].

[41] ATLAS collaboration, Search for R-parity-violating supersymmetric particles in multi-jet final states produced in p-p collisions at $\sqrt{s}=13 \mathrm{TeV}$ using the ATLAS detector at the LHC, Phys. Lett. B 785 (2018) 136 [arXiv:1804.03568] [InSPIRE].

[42] N. Bizot, G. Cacciapaglia and T. Flacke, Common exotic decays of top partners, JHEP 06 (2018) 065 [arXiv: 1803.00021] [INSPIRE].

[43] CDF collaboration, T. Aaltonen et al., Search for Pair Production of Strongly Interacting Particles Decaying to Pairs of Jets in p $\bar{p}$ Collisions at $\sqrt{s}=1.96$ TeV, Phys. Rev. Lett. 111 (2013) 031802 [arXiv: 1303.2699] [INSPIRE]. 